\title{
A prospective study of the psychological impact on patients with a first episode of genital herpes
}

\author{
Orla Carney, Emma Ross, Chris Bunker, George Ikkos, Adrian Mindel
}

\begin{abstract}
Objectives-To assess the psychological impact of first episode of genital herpes, and to determine whether this changes over time.

Setting and subjects-The Departments of Genitourinary Medicine (GUM), and Dermatology, Middlesex Hospital London. The study group consisted of patients attending the department of GUM with a clinically proven first episode of genital herpes. Two control groups were recruited; firstly patients without herpes attending the GUM Department and secondly patients attending the Dermatology Department out patients with chronic dermatoses.

Methods-Patients and controls completed an 87 item, self-administered psychological questionnaire at 3 monthly intervals for a year. The questionnaire consisted of the General Health Questionnaire (GHQ); the Hospital Anxiety and Depression Questionnaire (HADQ); Illness Attitude Scales and Illness Concern. Patients were also asked questions about their sexual behaviour.
\end{abstract}

Results-Ninety one patients (68 women, 23 men) with genital herpes, 61 GUM controls (42 women, 19 men) and 56 dermatology controls (36 women, 20 men) participated. There were no statistically significant demographic differences between patients and controls. At first visit the proportion of patients classified as "cases" by the GHQ (GHQ cases) were similar for primary herpes patients $62 \%(56 / 91)$ and Dermatology controls $52 \%$ (29/56) while a significantly smaller proportion of GUM controls $34 \%$ (21/61) were classified as GHQ cases. The primary herpes group were significantly more concerned about their illness than either the GUM controls or the Dermatology controls $(p<0.002)$. The proportion of primary herpes patients classified as "cases" by the GHQ reduced significantly over the initial three month period with $67 \%$ of patients classified as "cases" at their first visit becoming "noncases" after three months (p $<0.0001$ ). Also $50 \%$ of those classified as "cases" at first visit by the HADQ became "noncases" after the initial three months $(p=0 \cdot 007)$. The illness concern scores also decreased significantly from visit one to visit two (means $14 \cdot 7$ vs. $12 \cdot 3$; p < 0.0001).
Conclusion-The diagnosis of a first episode of genital herpes has a profound emotional effect on patients. If they do not have recurrent episodes, their emotional state improves. For those who do have recurrences, the level of anxiety and concern remains as high as at the time of their first diagnosis. Clinicians must be sensitive to the emotional impact such a diagnosis may bring.

(Genitourin Med 1994;70:40-45)

\section{Introduction}

Genital herpes is a common sexually transmitted infection. It is often recurrent and sometimes painful. Primary infections can be acute and debilitating, causing systemic illness as well as severe genital disease. Recurrent infection varies in frequency and severity, however, both primary and recurrent episodes are self limiting and cause no physical sequelae. There is, however, a wealth of research to suggest that there is a psychological morbidity associated with genital herpes. ${ }^{1-7}$ In 1981 Luby and Gillespie ${ }^{1}$ described a syndrome, akin to Kubler-Ross' stages of bereavement that patients may experience when they realise that they have genital herpes: denial, belief that there is a cure, realisation that they have herpes, loneliness, anger towards sexual partners, fear of sexual deprivation, and development of poor self image.

However, a mythology seems to have evolved around affect and recurrences, namely that negative mood directly reactivates the virus. Consequently, most previous work has concentrated on the cause and effect of recurrent infection. In one study, participants were asked to keep prospective diaries of stressful events, and to monitor their recurrence rate. They were then asked after the event what they felt had caused reactivation of the virus. "Retrospective reports almost unanimously identified stressful antecedents of herpes reactivation, yet concurrent assessment failed to confirm this". ${ }^{3}$ As Kemeney et al have said "the association found between depressive mood and herpes recurrences may be attributable to the feeling of depression that results from having a high rate of recurrences". ${ }^{4}$ One may surmise that, because of the capricious nature of the infection, such retrospective reasoning results from an attempt to have some control, albeit tenuous, over the virus.

Little work has been done on what impact \\ 13 October 1993 \\ The Academic
Department of
Genitourinary
Medicine, University
College and Middlese
School of Medicine,
James Pringle House,
The Middlesex
Hospital, London
W1N 8AA
O Carney
E Ross
A Mindel
Department of
Psychiatry, Middlesex
Hospital, London
G Ikkos
Department of
Dermatology,
Middlesex Hospital,
London
C Bunker
Address correspondence to:
Professor A Mindel,
Academic Unit of Sexual
Health Medicine, Sydney
Hospital, GPO Box 1614,
Sydney 2001, Australia.
Accepted for publication
13 October 993
}


the first episode of herpes has on the individual. It is not therefore known whether there is psychological morbidity associated with a first episode that is absent in the case of other sexually transmitted infections. If such a morbidity were found to exist it might alter over time, or be dependent on whether the patient has recurrences or not. Conversely, such a morbidity might be used as a predictor for the physical pattern of the disease, if as is suggested by Goldmeier et $a l^{7-9}$ the emotional state of the patient helps to trigger recurrences.

We therefore designed a study with the following aims: (1) To assess the psychological morbidity of patients with a first episode of genital herpes infection, (2) To monitor whether this changes over time, (3) To assess whether subsequent episodes had any bearing on adjustment to the illness.

\section{Methods}

Between March 1988 and October 1990, all patients who presented with a first episode of genital herpes to the department of Genitourinary Medicine of the Middlesex Hospital, London, were invited to take part in the study at their follow up visit, 7-10 days after initial presentation. This was to ensure that the diagnosis had been confirmed and fully discussed with the patient according to clinic protocol.

There were two control groups: (1) New patients of the same sex presenting to the GUM department on the same day as the index case, but who did not have herpes (GUM controls), (2) Patients with chronic dermatoses attending as out-patients at the Middlesex Hospital (dermatology controls).

The former group was included because previous studies have suggested that there is a higher psychiatric morbidity in patients attending genitourinary clinics than in the general population. ${ }^{10-14}$ Because there is also work to suggest that there is a psychological morbidity associated with both chronic illness, and skin disorders, ${ }^{15-21}$ a second control group was included, which was comprised of patients of roughly the same age as those attending the GUM clinic, who had a chronic, recurrent, but not sexually transmitted, disorder. Informed consent was obtained from all participants.

\section{Questionnaire}

Participants were asked to complete an 80 item, self administered questionnaire, divided into four sections, each consisting of a well established psychological questionnaire: (1) General Health Questionnaire (GHQ) (30 items), (2) Hospital Anxiety and Depression (HAD) (14 items), (3) Illness Attitude Scales (IAS) (27 items), (4) Illness concern (nine items).

The GHQ measures non psychotic psychiatric illness. In this, responses are only counted as being present if the patient considers that they represent a deviation from their own norm. The 30 item inventory was used, and "caseness" was defined as a score of five or more in keeping with previous studies. ${ }^{22}{ }^{23}$ However, some of the responses may be influenced by concurrent physical illness. To control for this we also used the HAD inventory. ${ }^{24}{ }^{25}$ This is said to be unbiased by coexisting physical illness, since it avoids questions which may have a somatic cause. Case definition is taken as a score of eight or more on either of the anxiety or depression subscales. For the purposes of analysis, GHQ and HAD scores were treated as dichotomous variables (case or non case) using the cut-off points described above.

The IAS measure fears, beliefs and attitudes associated with hypochondriasis and abnormal illness behaviour. Characteristics of other psychiatric syndromes are excluded. ${ }^{26}$ There are nine scales, each consisting of three questions. The IAS is scored from $0-12$.

Conversely, illness concern assesses the actual and potential emotional impact caused by the specific problem which has brought them to the clinic, and scores range from 2-27.

Seven questions were also asked, about sexual orientation, numbers of sexual partners, and frequency and enjoyment of sex.

In addition, those with herpes were asked questions on their basic knowledge of the virus. The rationale for this was to ascertain whether misconceptions about genital herpes would lead to increased anxiety, compared to those who were more informed about the subject. Patients and controls were asked to repeat the questionnaire at three monthly intervals for a year.

\section{Analysis}

Poor follow-up rates meant that statistical comparisons were confined to the assessments of the three groups at their first visit (to establish whether primary HSV has a substantial effect on patients) and to the first two assessments (to establish whether there were any changes in the outlook of these patients between their initial visit and their first 3 month follow-up attendance).

Within the primary HSV group, patients who had recurrences at visit two were compared with those who did not have recurrences to establish whether recurrences were an influential factor in determining the patients psychological state.

Analysis of variance (ANOVA) was used to test for any difference in the age distributions of the groups of patients, and the Chi square test was used to test for evidence of an association between the dichotomous variables ("case"/"non-case") and patient group. The distributions of the data for the three groups were compared using the Kruskal-Wallis ANOVA by ranks. ${ }^{27}$ Comparison of these distributions in any two groups were made using the Mann-Whitney U statistic. To investigate any difference in patients attitudes from visit one to visit two McNemar's test for changes in proportion and Wilcoxon's test for comparing scores were applied. ${ }^{27}$ 


\section{Results}

Patients ( $n=195$ ) were initially identified as having primary genital herpes, and 91 entered the study. Of the remaining 104, 65 failed to keep their follow up appointment, 26 initially

Table 1 Demographic characteristics of the three groups

\begin{tabular}{llll}
\hline & $\begin{array}{l}\text { Genital herpes } \\
\text { group } \\
n=91\end{array}$ & $\begin{array}{l}\text { Genitourinary } \\
\text { controls } \\
n=61\end{array}$ & $\begin{array}{l}\text { Dermatology } \\
\text { controls } \\
n=56\end{array}$ \\
\hline $\begin{array}{l}\text { Age Mean (s.d.) } \\
\text { (years) }\end{array}$ & $28 \cdot 3$ & $26 \cdot 3(7 \cdot 72)$ & $27 \cdot 6(7 \cdot 72)$ \\
$\begin{array}{l}\text { Sex: number (\%) } \\
\text { Male }\end{array}$ & $(8 \cdot 14)$ & & \\
$\quad$ Female & $23(25)$ & $54(90)$ & $54(96)$ \\
$\begin{array}{l}\text { Sexual } \\
\text { orientation: number (\%) }\end{array}$ & $68(75)$ & $6(10)$ & $2(4)$ \\
$\quad$ Heterosexual & $86(96)$ & $52(90)$ & $45(96)$ \\
$\quad$ Homosexual & $4(4)$ & $6(10)$ & $2(4)$ \\
$\begin{array}{l}\text { Employed: number (\%) } \\
\text { Civil status: number (\%) }\end{array}$ & $82(96)$ & $49(90)$ & $44(81)$ \\
$\quad$ Single & $56(62)$ & $43(72)$ & $40(74)$ \\
Cohabiting/married & $24(27)$ & $15(25)$ & $12(22)$ \\
Separated/divorced & $10(11)$ & $2(3)$ & $2(4)$ \\
\hline
\end{tabular}

GUM-genitourinary medicine.

^Excluding 10 patients whose sexual orientation was unknown.

Table 2 Number of respondents through the study

\begin{tabular}{llll}
\hline Time & $\begin{array}{l}\text { Genital } \\
\text { herpes group }\end{array}$ & $\begin{array}{l}\text { Genitourinary } \\
\text { controls }\end{array}$ & $\begin{array}{l}\text { Dermatology } \\
\text { controls }\end{array}$ \\
\hline Initial visit & 91 & 61 & 56 \\
After 3 months & $61(67 \%)$ & $30(49 \%)$ & $34(61 \%)$ \\
After 6 months & $51(56 \%)$ & $21(34 \%)$ & $27(48 \%)$ \\
After 9 months & $40(44 \%)$ & $17(28 \%)$ & $20(36 \%)$ \\
After 12 months & $28(31 \%)$ & $11(18 \%)$ & $18(32 \%)$ \\
\hline
\end{tabular}

^Number (\%) at each follow up visit.

Table 3 General Health Questionaire (GHQ)-number and percentage of cases at each visit

\begin{tabular}{|c|c|c|c|}
\hline Visit number & $\begin{array}{l}\text { Genital } \\
\text { herpes group }\end{array}$ & $\begin{array}{l}\text { Genitourinary } \\
\text { controls }\end{array}$ & $\begin{array}{l}\text { Dermatology } \\
\text { controls }\end{array}$ \\
\hline $\begin{array}{l}\text { Visit } 1^{\star} \\
\text { Visit } 2^{\star} \\
\text { Visit } 3^{\star} \\
\text { Visit } 4^{\star} \\
\text { Visit } 5^{\star}\end{array}$ & $\begin{array}{r}56 / 91(62) \\
18 / 61(30) \\
14 / 51(27) \\
14 / 40(35) \\
7 / 28(25)\end{array}$ & $\begin{array}{l}21 / 61(34) \\
6 / 30(20) \\
4 / 21(19) \\
6 / 17(35) \\
5 / 11(45)\end{array}$ & $\begin{array}{r}29 / 56(52) \\
10 / 34(29) \\
11 / 27(41) \\
7 / 20(35) \\
7 / 18(39)\end{array}$ \\
\hline
\end{tabular}

^Number of GHQ “cases”/number of patients (\%).

Table 4 Hospital Anxiety and Depression (HAD)—number and percentage of anxiety "cases" at each visit

\begin{tabular}{llll}
\hline Visit number & $\begin{array}{l}\text { Genital } \\
\text { herpes group }\end{array}$ & $\begin{array}{l}\text { Genitourinary } \\
\text { controls }\end{array}$ & $\begin{array}{l}\text { Dermatology } \\
\text { controls }\end{array}$ \\
\hline Visit $1^{\star}$ & $47 / 91(52)$ & $22 / 61(36)$ & $22 / 56(34)$ \\
Visit 2 & $21 / 61(34)$ & $7 / 30(23)$ & $13 / 34(38)$ \\
Visit 3* & $15 / 51(29)$ & $6 / 21(29)$ & $12 / 27(44)$ \\
Visit 4 & $17 / 40(42)$ & $4 / 17(23)$ & $6 / 20(30)$ \\
Visit 5* & $12 / 28(43)$ & $4 / 11(36)$ & $8 / 18(44)$ \\
\hline
\end{tabular}

^Number of HAD anxiety "cases"/number of patients (\%).

Table 5 Hospital Anxiety and Depression (HAD)—number and percentage of depression "cases" at each visit

\begin{tabular}{llll}
\hline Visit Number & $\begin{array}{l}\text { Genital } \\
\text { herpes group }\end{array}$ & $\begin{array}{l}\text { Genitourinary } \\
\text { controls* }\end{array}$ & $\begin{array}{l}\text { Dermatology } \\
\text { controls* }\end{array}$ \\
\hline Visit 1 & $15 / 91(16)$ & $3 / 61(5)$ & $7 / 56(12)$ \\
Visit 2 & $3 / 61(5)$ & $3 / 30(10)$ & $2 / 34(6)$ \\
Visit 3 & $4 / 51(8)$ & $1 / 21(5)$ & $2 / 27 \cdot(7)$ \\
Visit 4 & $3 / 40(8)$ & $3 / 17(18)$ & $2 / 20(10)$ \\
Visit 5 & $2 / 28(7)$ & $0 / 11(0)$ & $2 / 18(11)$ \\
\hline
\end{tabular}

^Number of HAD anxiety "cases"/number of patients (\%). agreed to participate but then failed to return the questionnaire, 10 had a history of recurrent genital herpes, and three were diagnosed as having primary syphilis and not genital herpes. In addition, 61 GUM controls and 56 dermatology controls were recruited.

Of the 61 GUM controls, 50 had genital tract infections including eight with multiple infections. Eleven patients were found to have no infection, or a non sexually transmitted problem such as a urinary tract infection. Three of the GUM control group had HIV antibody tests, all of which were negative.

Table 1 shows the demographic characteristics of the three groups. There were no statistically significant differences between the three groups with respect to the proportions of males and females or to their age distributions. Table 2 shows the number of respondents from all three groups followed up over the year.

General Health Questionnaire (table 3)

At first visit, $62 \%$ of those with a first episode of genital herpes could be classified as GHQ "cases" compared with $52 \%$ of patients attending the dermatology clinic and $34 \%$ of GUM controls $(p<0.005)$. This significant difference between the three groups was mostly attributable to a difference between the primary HSV patients and the GUM controls $(p=0.002)$. By the second visit, there was a marked decrease in the proportion of patients with herpes classified as GHQ cases. McNemar's test for changes in proportion showed that $67 \%$ of those who were classified as "cases" at visit one became "noncases" by their second visit $(p<0.001)$. There were no significant changes from visit one to visit two for either the GUM or dermatology controls.

\section{Anxiety (table 4)}

There were no significant differences in the proportion of anxiety "cases" in the three groups at visit 1 . Within the primary HSV group $50 \%$ of patients classified as anxiety "cases" at first visit became "noncases" by their second visit $(p=0.007)$. There were no changes from visit one to visit two for the other two groups.

\section{Depression (table 5)}

No significant differences were observed between the three groups at first visit and no changes were noted within the groups over time.

\section{Illness Attitude Scales}

At first visit, the distributions of pain scores in the three groups were significantly different (means for primary herpes, GUM controls, and dermatology controls were $4.9,4.4$ and 3.5 respectively, Kruskal Wallis $\mathrm{p}<0.004$ ). Subsequent analysis by the Mann-Whitney test indicated that HSV patients and GUM controls were in more pain than dermatology patients $(p<0.002$ and $p<0.04$ respectively). There was no significant difference in the pain scores of the primary HSV and GUM attenders. 
Figure 1 Mean illness concern at each visit comparing patients with primary genital herpes, genitourinary controls, and patients with chronic patients with chronic dermatoses. All three groups showed a decrease. decrease was only significantly different in the herpes group $(p<0.0001$ comparing visits 1 and 2 ).

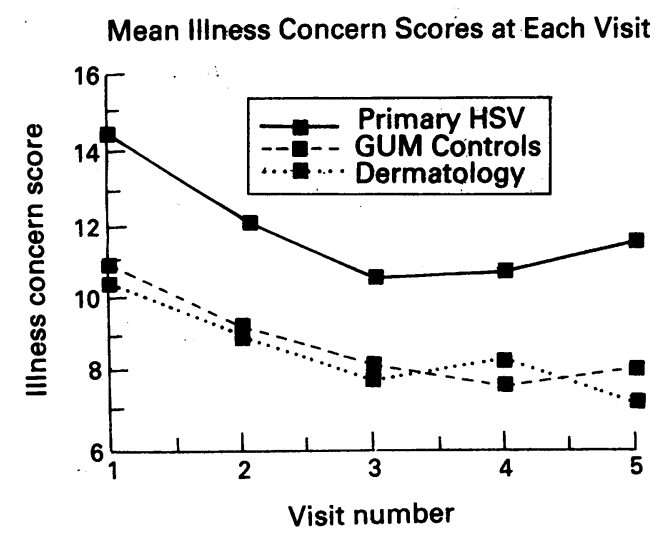

With respect to their treatment experience the three groups were also different (means for primary HSV, GUM controls, and dermatology controls were $4.9,5.8$, and 6.4 respectively Kruskal Wallis $p=0.0001$ ). The Mann-Whitney test suggested the dermatology group scored significantly higher than the primary HSV patients $(\mathrm{p}<0.0001)$ and the GUM controls scoring higher than the patients with primary HSV $(p=0.013)$. The dermatology patients scored higher than GUM patients $(p=0.033)$.

From visit one to visit two there was no statistically significant change in the scores within each of the three groups.

\section{Illness Concena (figure)}

At first visit there was a statistically significant difference between the scores of the three groups (means for primary HSV, GUM controls, dermatology controls were 14.4 , 10.4 , and 10.9 respectively Kruskal Wallis $\mathrm{p}<0.0001$ ). Subsequent Mann-Whitney tests indicated that the primary HSV group were more concerned about their illness than either GUM controls or dermatology patients $(p<0.0001, p<0.002$, respectively). Primary HSV patients became less concerned at visit two compared to visit one (mean 12.3 vs $14.7 ; \mathrm{p}<0.0001$ ).

\section{Sexual behaviour}

No statistically significant differences were found between the study group and the controls with respect to the quality or frequency of their sexual intercourse.

\section{Herpes knowledge}

No association was found between herpes knowledge scores and scores in other sections of the questionnaire. However, women did score consistently higher than men.

\section{Recurrences of herpes}

Of the 61 herpes patients who completed the second questionnaire 26 (six male, 20 female) had had at least one recurrence, whilst 34 (nine male, 25 female) had none. Bata were missing on one female patient. Selecting those patients who had no recurrence within the first three months, and comparing their scores at visit one and visit two, the Wilcoxon test showed their Illness Concern scores to be significantly lower at visit two than at visit one (mean 12.3 vs $15.8, \mathrm{p}=0.0003$ ). McNemar's test showed a change in the proportion of GHQ cases with $73 \%$ who were initially classified as "cases" being classified as "noncases" three months later ( $p<$ $0.0001)$. The same pattern was observed amongst anxiety "cases" with $55 \%$ of those classified as "cases", at visit one becoming "noncases" at visit two $(p=0.006)$. Those patients who did have recurrences remained as concerned at visit two as at visit one, and there were no significant changes in the proportion defined as cases at either visit $(13 / 26$ $(50 \%)$ at visit one vs $11 / 26(31 \%)$ at visit two). Taking all 60 primary herpes patients who were followed up to visit two, $58 \%$ $(15 / 26)$ of those known to have had recurrences were defined to be GHQ cases at visit one versus $76 \%(26 / 34)$ of those who did not experience recurrences. The respective figures for ANX cases were $50 \%(13 / 26)$ versus $59 \%(20 / 34)$. At visit two, 38\% (10/26) of those with recurrences were defined as GHQ cases versus $24 \%(8 / 34)$ of those with no recurrences. The figures for ANX cases at visit two were $42 \%(11 / 26)$ and $29 \%(10 / 34)$ respectively ( $p>0.2$ in each case).

\section{Discussion}

The majority of previous studies on the psychological impact of genital herpes have concentrated on the cause and effect of recurrent infection. Little has been written on the psychological morbidity of those who present with a first episode of genital herpes, or on whether this has any bearing on the subsequent disease pattern, and vice versa. Previous studies have suggested that those with a high GHQ score at the time of their first episode are likely to experience recurrences sooner than those with low scores (7-9), the suggestion being that the patient's emotional state has a direct impact on reactivation of the virus. However, our study suggests that, whilst $62 \%$ of patients with genital herpes could be classified as GHQ "cases" at the time of the first episode only those with recurrences tended to continue as "cases", whilst those who did not experience recurrences were likely to become "noncases". This same pattern was seen amongst anxiety "cases", and in a decrease in their Illness Concern scores from visit one to visit two.

However, our study does not allow direct comparison with the studies mentioned above, ${ }^{7-9}$ because it was designed very differently. Goldmeier et al in $1988^{\circ}$ studied all genital herpes patients presenting to an STD clinic. Fifty seven patients with first episode genital herpes were compared with 50 patients with frequently recurring herpes. GHQ scores were statistically higher in those who presented with recurrent genital herpes. Our study took only first episode genital herpes and assessed them as recurrent or non recurrent at their second visit (three months after entry). It may be that patients with many recurrences have more psychological morbidity than those presenting with the first 
episode. Goldmeier and Johnson ${ }^{7}$ analysed the role of recurrences in primary HSV patients by survival analysis, looking at whether it was higher in patients with preexisting psychiatric illness. They were able to do this as they took the date of the primary attack to the date a patient presented with a recurrent episode within 30 weeks of their date of entry. Patients who did not present were assumed to have had no recurrences. Our patients, however, were requested to return every 3 months and complete a questionnaire, whether or not they had symptoms, and recurrences were therefore all noted at the same time points. The conclusion reached by Goldmeier and Johnson was that recurrence rates in those with GHQ scores $\geqslant 12$ were higher than in those with scores $\leqslant 11$. Poor follow up in our study did not allow for a similar assessment.

It would seem from the results in the present study that a first episode of genital herpes does place a severe emotional burden on patients, but that this may lessen over time if they do not experience recurrences. However, with only $50 \%$ of patients initially diagnosed as primary genital herpes entering the study, patient self selection may have influenced the results, and generalisations to the majority of patients with herpes should be made with caution.

At first visit we would have expected to find more acute anxiety and depression, as designated by the HAD questionnaire. However, with the high scores at first visit for Illness Concern, it would seem that it is genital herpes which is the focus of their emotional concerns. Given that Illness Concern also measures potential worries regarding their illness, and for those who do not have any recurrences within the first three months their scores drop, we could extrapolate that it is the physical reality of the actual blisters and sores which causes emotional problems.

Basic knowledge about the virus did not seem to be associated with low scores on any section of the questionnaire or identification as a "case" on the GHQ or HAD questionnaire, reiterating the adage that health education does not necessarily lead to behavioural or attitudinal change.

What is also interesting is that, as a group, those with a first episode of genital herpes would seem to have a higher incidence of psychiatric morbidity than other patients attending a genitourinary clinic for the first time, and those with chronic dermatoses attending as dermatology out-patients on a regular basis. Previous studies have shown that $30-40 \%$ of GUM attenders can be classified as GHQ "cases". 112 This compares with $34 \%$ in our study population. Using the HAD inventory, Barczak et $a l^{24}$ found the prevalence of psychiatric disorder within the GUM clinic to be $31 \%$, whereas we found it to be approximately $36 \%$, suggesting that this control group was similar to those in previous studies. It is rather those with a first episode of herpes who seemed to have a higher psychiatric morbidity $(62 \%$ classified as GHQ cases and $52 \%$ as HAD anxiety cases) than the already high morbidity of those attending GUM clinics.

Much work on emotional problems relating to skin disorders has been anecdotal, suggesting that those who have dermatological problems have high levels of anxiety, guilt and shame, and lack self confidence. ${ }^{17}$ Wessely and Lewis found that, using the 12 item inventory, $42 \cdot 7 \%$ of dermatology patients could be classified as GHQ "cases". ${ }^{17}$ This compares with $52 \%(29 / 56)$ at first visit, which fell to $39 \%(7 / 18)$ at final visit, in the present study population. In 1983, Hughes et $a l^{20}$ found that using the 30 item GHQ inventory, $30 \%$ of dermatology out patients could be classified as GHQ "cases". In addition, those who had visible skin lesions, on hands or the face, were found to have higher scores than those with less visible conditions. This last point is interesting, given the intimate nature of the majority of genital herpes lesions.

We would suggest that we cannot determine who will have recurrences from their psychological profile at first visit. Recurrences may be independent of mood per se, but in themselves can have a damaging effect on the individual's well-being. The severity of problem specific scores compared with those of the two control groups, and the lower scores for general states would seem to underline this and appropriate counselling should always be considered.

Clinicians need to acknowledge that not only may patients with a first episode of genital herpes be experiencing an acute and debilitating physical illness, but also that its diagnosis may precipitate further emotional reactions.

1 Luby ED, Gillespie O. Psychological responses to genital herpes. Helper 1981;3:2-3.

Manne S, Sandler I. Coping and adjustment to genital herpes. F Behav Med 1984;7:391-410.

3 Vanderplate C, Aral S. Psychosocial aspects of genital herpes virus infection. Health Psychol 1987;6:57-72.

4 Kemeny $M$, Cohen $F$, Zegans $L$, Conant $M$. Psychological and immunological predictors of genital herpes recurrence. Psychosom Med 1989;51:195-208.

5 Luby E, Klinge V. Genital herpes: a pervasive psychosocial disorder. Arch Dermatol 1985;121:494-7.

6 Rand K, Hoon EF, Massey JK, Johnson JH. Daily stress and recurrence of genital herpes simplex. Arch Intern Med 1990;150:1889-93.

7 Goldmeier D, Johnson A. Does psychiatric illness affect the recurrence rate of genital herpes? $\mathrm{Br} \mathcal{F}$ Venereal Dis 1982;58:40-3.

8 Goldmeier D, Johnson A, Jeffries D, Walker GD, Underhill G, Robinson G, Ribbans H. Psychological aspects of recurrences of genital herpes. $\mathcal{f}$ Psychosom Res aspects of recurre

9 Goldmeier D, Johnson A, Byrne M, Barton S. Psychosocial implications of recurrent genital herpes simplex virus infection. Genitourin Med 1988;64: 327-30.

10 Wells BWP. Personality study of VD patients. $\mathrm{Br} \mathcal{F}$ Venereal Dis 1970;46:498-501.

11 Pedder JR, Goldberg DP. A survey by questionnaire of psychiatric disturbance in patients attending a venereal disease clinic. BrF Venereal Dis 1970;40:56-61.

12 Mayou R. Psychological morbidity in a clinic for sexually transmitted disease. Br 7 Venereal Dis 1975;51:57-60.

13 Catalan J, Bradley M, Gallwey J, Hawton K, Sexual dysfunction and psychiatric morbidity in patients attending function and psychiatric morbidity in patients attending a clinic for sexually

14 Fulford WM, Catterall RD, Hoinville E, Lim KS, Wilson $D$. Social and psychological factors in the distribution of STD in male clinic attenders. Br $\mathcal{F}$ Venereal Dis 1983; 59:381-5. 
15 Wessely SC, Lewis GH. The classification of psychiatric morbidity in attenders at a dermatology clinic. $\mathrm{Br} \mathcal{F}$ Psychiatry 1989;155:686-91.

16 Logan RA. Self help groups for patients with chronic skin diseases. Br 7 Dermatol 1988;118:505-8.

17 Cotterill JA. Psychiatry and the skin. Br $\mathcal{F}$ Hosp Med 1989;42:401-4.

18 Beaman K, Luzzatto P. Psychological approaches to the treatment of skin diseases. Nursing 1988;29:1061-3.

19 Hardy GE, Cotterill JA. A study of depression and obessionality in dysmorphophobic and psoriatic
ond obessionality in dysmorphophobic

20 Hughes JE, Barraclough BM, Hamblin LG, White JE Psychiatric symptoms in dermatology patients. $B r \dot{f}$ Psychiatry 1983;143:51-4.

21 Bombardier CH, D'Amico C, Jordan JS. The relationship of appraisal and coping to chronic illness adjustment.
Behav Res Ther 1990;28:297-304.

22 Goldberg DP. The detection of psychiatric illness by questionnaire. Maudsley Monographs (OUP) 1972; No. 21.

23 Benjamin S, Decalmer $P$, Haran $D$. Community screening for mental illness: a validity study of the general health questionnaire. Br f Psychiatry 1982;140:174-80.

24 Barczak P, Kane N, Andrews S, Congdon AM, Clay JC, Betts T. Patterns of psychiatric morbidity in a genitourinary clinic. Br ₹ Psychiatry 1988;152:698-700.

25 Lewis G, Wessely S. Comparison of the general health questionnaire and the hospital anxiety and depression queale. Br $¥$ Psychiatry 1990;157:860-4.

26 Kellner R. Abridged Manual of the Ilness Attitude Scale. Department of psychology school of medicine. University of New Mexico. 1986.

27 Siegel S. Non-parametric statistics for the behavioural sciences. McGraw-Hill, New York. 1956. 